\title{
The Association between Physical Exercise and Reactive Oxygen Species (ROS) Production
}

\author{
Albená Nunes-Silva ${ }^{1,3}$ and Leandro Ceotto Freitas-Lima ${ }^{2,3}$ \\ ${ }^{1}$ Departamento Morfologia, Laboratório de Resolução da Resposta Inflamatória, Brazil \\ ${ }^{2}$ Departamento de Fisiologia e Biofísica, Brazil \\ 3 Instituto de Ciências Biológicas - Universidade Federal de Minas Gerais, Belo Horizonte, Brazil
}

*Corresponding author: Albená Nunes da Silva, Departamento Morfologia, Laboratório de Resolução da Resposta Inflamatória, Instituto de Ciências Biológicas, Universidade Federal de Minas Gerais, Belo Horizonte, Brazil, Tel: 5531 9992-3426; E-mail: albenanunes@hotmail.com

Rec date: Aug 07, 2014, Acc date: Nov 25, 2014, Pub date: Nov 27, 2014

Copyright: (c) 2015 Nunes-Silva A, et al. This is an open-access article distributed under the terms of the Creative Commons Attribution License, which permits unrestricted use, distribution, and reproduction in any medium, provided the original author and source are credited.

\begin{abstract}
The importance of exercise has been widely accepted by the public, professional organizations and the medical community, and the number of people around the world who practice day-to-day regular physical activity has recently increased. Physical activity is a primary requirement for the maintenance and promotion of health. However, intense exercise induces an increase in substrate utilization by the working muscles, resulting in an increased use of oxygen. This rise in oxygen consumption combined with the activation of specific metabolic pathways during or after exercise results in the generation of Reactive Oxygen Species (ROS). These substances are produced naturally by oxidative metabolic processes and are highly reactive. ROS can be useful in some situations; for example, macrophages use hydrogen peroxide to destroy bacteria. ROS increases during high-intensity exercise and its production is related to a large number of diseases, such as emphysema, inflammatory diseases, atherosclerosis, cancer and aging. During evolution, living organs developed an endogenous mechanism to minimize the damage caused by ROS, termed the antioxidant defense system. The imbalance between the production of ROS and the removal of these compounds by the antioxidant defense system causes a condition known as oxidative stress. This interesting paradox is exemplified by the hypothesis that physical exercises known to promote appropriate standards of health are also related to increased ROS production, which in turn closely correlate with various diseases. Recent publications have shown that ROS produced by exercise in adequate amounts may be linked to exercise benefits, both for health and for athletic performance. Thus, the aim of this article is to examine the close association between physical exercise and the production of ROS through an extensive literature review.
\end{abstract}

Keywords: Physical exercise; Oxidative stress; Reactive oxygen species; Health

$$
\begin{aligned}
& \text { Abbreviations } \\
& \text { Anti-PECAM-1 = Antibody anti } \\
& \text { molecule- } 1 \\
& \mathrm{CAT}=\text { Catalase } \\
& \mathrm{C} 57 \mathrm{Bl} / 6 \text { = Wild-type mice } \\
& \mathrm{COX} 2=\text { Cyclooxygenase- } 2 \\
& \mathrm{ETC}=\text { Electron transport chains } \\
& \mathrm{GPx}=\text { Glutathione peroxidase } \\
& \mathrm{GR}=\text { Glutathione reductase } \\
& \mathrm{GSH}=\text { Reduced glutathione } \\
& \mathrm{GSSG}=\text { Oxidized glutathione } \\
& \mathrm{OH} \bullet=\text { Hydroxyl radical } \\
& \mathrm{H}_{2} \mathrm{O}_{2}=\text { Hydrogen peroxide } \\
& \mathrm{MDA}=\text { Malonaldehyde } \\
& \mathrm{mA}=\text { Miliampere }
\end{aligned}
$$

Anti-PECAM-1 = Antibody anti platelet endothelial cell adhesion
NADPH oxidase $=$ Nicotinamide adenine dinucleotide phosphateoxidase

$$
\begin{aligned}
& { }^{1} \mathrm{O}_{2}=\text { Singlet oxygen } \\
& \mathrm{O}_{2}=\text { Molecular oxygen } \\
& \mathrm{O}_{2} \bullet-=\text { Anion superoxide radical }
\end{aligned}
$$

RT-PCR = Real Time polymerase chain reaction.

ROS $=$ Reactive oxygen species

$\mathrm{RLU}=$ Relative light unit

SOD $=$ Superoxide dismutase

TBA $=$ Thiobarbituric acid

TBARS $=$ Thiobarbituric acid reactive substances

$\mathrm{XO}=$ Xanthine oxidase

\section{Introduction}

Since the beginning of history, philosophers and health professionals have written manuscripts noting that regular exercise is an essential part of a healthy lifestyle. Over 2.400 years ago, the Greek philosopher Hippocrates described [1]: 
"Just take care about eating will not keep a man health, he should also do exercise, once the food and exercise, work together to produce health." [1]

Since Hippocrates time, the health benefits of physical exercise have become well known, including a reduced risk for serious problems related to overweight/obesity and physical inactivity and improvement in cardio respiratory function and physical abilities such as strength, flexibility, endurance and muscle power performance. The importance of exercise has achieved wide acceptance from professional health organizations and the medical community. Currently, people around the world make exercise a common, routine activity.

However, exercise increases substrate utilization by worked muscles due to the increase in energy demand and the use of oxygen required to produce energy through the oxidative pathway, which can be a dangerous process. This increase in molecular oxygen $\left(\mathrm{O}_{2}\right)$ consumption combined with the activation of specific metabolic pathways during and after exercise can result in ROS formation. Over the past 30 years, studies have examined ROS production during and after exercise [2-12]. This manuscript describes the interaction between ROS and physical exercise.

ROS are also known as free radicals. A free radical is defined as "any atom, molecule or molecule fragment containing one or more unpaired electrons in their valence layers" [13]. ROS are produced naturally by oxidative metabolic processes and are highly reactive with a half-life in the order of milliseconds. They are often extremely useful, such as in situations where there is a need for the activation of the immune system. For example, macrophages use $\mathrm{H}_{2} \mathrm{O}_{2}$ to destroy bacteria and other foreign elements [13].

ROS increase after high-intensity exercise and are related to a large number of diseases, such as emphysema, inflammatory diseases, atherosclerosis, cancer and aging [14-17]. Physical exercise is a wellestablished procedure to protect the body against risk factors; however, the relationship between exercise and molecules that are detrimental to body integrity is not fully understood.

To maintain life in ambient air during evolution, a sophisticated endogenous mechanism termed the antioxidant defense system that minimized damage caused by ROS was created [13]. The imbalance between the production of ROS and the removal of these compounds by the antioxidant defense system causes a condition known as oxidative stress [13]. Oxidative stress due to a high concentration of ROS causes molecular damage to cellular structures, with consequent impairment of tissue or organ functions $[13,15,16]$. Additionally, ROS can lead to RNA and DNA mutation, thereby causing changes in cellular function and tissue death $[13,15,16]$. This oxidative damage can be visualized in various organs and tissues, such as skeletal muscle, cardiac muscle, liver, fat tissue, brain and the vascular system [17]. The major mechanism of injury induced by ROS is called lipid peroxidation and pertains to the oxidation of the lipid layer of the cell membrane [13]. However, the deleterious effects of oxidative stress vary considerably between living organs according to age, physiological state and diet status $[13,15,16]$.

There is little doubt that the practice of moderate exercise is effective for prolonging life and reducing mortality $[18,19,20]$. However, studies have shown that submaximal exercise also results in significant physiological and immunological changes in susceptibility of red blood cells to osmotic and oxidative stress [21,22]. The specialized scientific literature shows evidence of the correlation between high intensity exercise and excess production of free radicals
[23-25]. High intensity exercise is defined as exercise in which oxygen consumption exceeds $70 \%$ of maximum oxygen consumption $\left(\mathrm{VO}_{2}\right.$ max.). Furthermore, exercise performed until fatigue sets in leads to increased use of the oxidative pathway of energy production and the production of ROS, causing an acute oxidative stress profile [15]. Thus, the aim of this article is to discuss the correlation between physical exercise and the production of ROS through an extensive literature review. Here we try to show that this relationship needs to be reviewed and this association is not just detrimental.

\section{Methods}

\section{Theoretical background}

The literature investigation utilized scientific databases including Pubmed, Bireme and Scielo by employing the following keywords: "exercise", "ROS", "oxidative stress", "anti-oxidant", and "supplementation". This search strategy returned more than 300 papers, of which approximately 60 contributed directly to this review.

\section{The paradox of oxygen}

(...) On the one hand, the use of oxygen through the monetization of energy substrates is favorable to the existence of complex life on Earth, on the other, has proved disastrous for the beings that use it (...) [13]

It is widely believed that the appearance of the first organisms capable of photosynthesis promoted a large modification in the atmosphere of prehistoric Earth. This atmosphere had a very different composition from the present atmosphere, containing large amounts of carbon dioxide $\left(\mathrm{CO}_{2}\right)$, nitrogen $\left(\mathrm{N}_{2}\right)$, water vapor $\left(\mathrm{H}_{2} \mathrm{O}\right)$, ammonia and possibly methane; despite these differences, ROS were still present [13]. Photosynthetic organisms used water vapor and light energy from the sun, converting it to chemical energy through the production of carbohydrates and producing $\mathrm{O}_{2}$ [13]. The use of oxygen in metabolic processes enabled the evolution and differentiation of simple single-celled organisms into complex current species including humans. The use of oxygen through the conversion of energy substrates is favorable for the existence of complex life on Earth; however, this use has proved disastrous for the beings that use it by damaging the cellular and subcellular structures of aerobic organisms [13]. Between $2-5 \%$ of all oxygen consumed by aerobiontes becomes ROS or free radicals that have a tendency to react with adjacent organic structures. These reactions alter organic structures, consequently modifying their functions and causing irreparable damage to the organ [17].

The group of free radicals includes the anion superoxide radical $\left(\mathrm{O}_{2} \bullet-\right)$ and the hydroxyl radical $(\mathrm{OH} \bullet)$. These molecules are derived from the metabolism of oxygen and are known ROS molecules. Other examples include $\mathrm{H}_{2} \mathrm{O}_{2}$ and singlet oxygen $\left({ }^{1} \mathrm{O}_{2}\right)$ [13].

\section{Formation of ROS}

ROS are formed during normal metabolism, including both enzymatic and non-enzymatic processes. Due to its configuration, oxygen tends to receive one electron at a time, enabling formation of highly reactive intermediates such as $\mathrm{O}_{2} \bullet-, \mathrm{H}_{2} \mathrm{O}_{2}$, and $\mathrm{OH} \bullet$ [13].

$\mathrm{O}_{2} \cdot$ - can be formed by univalent reduction of $\mathrm{O}_{2}$ in the mitochondrial electron transport chain by the complex multicomponent enzyme NADPH oxidase; however, unlike most free 
radicals $\mathrm{O}_{2} \bullet$ - is inactive [26]. In aqueous media the primary reaction is dismutation, which produces a molecule of $\mathrm{H}_{2} \mathrm{O}_{2}$ and an oxygen molecule $\left(\mathrm{O}_{2}\right)$. In the human body, $\mathrm{O}_{2} \bullet$ - can be eliminated by the enzyme SOD, which catalyzes the dismutation of two molecules of $\mathrm{O}_{2} \bullet$ - into oxygen $\left(\mathrm{O}_{2}\right)$ and $\mathrm{H}_{2} \mathrm{O}_{2}$. If not eliminated from the body by the family of antioxidant enzymes GPx and CAT, this molecule can generate $\mathrm{OH} \bullet \mathrm{ROS}$ and cause harmful effects (e.g., lipid peroxidation). The radical $\mathrm{O}_{2} \bullet$ - is of vital importance to defense cells; without it, the body is unprotected against infections caused by viruses, bacteria and fungi [13]. It is generated in vivo by phagocytes, lymphocytes and fibroblasts during the inflammatory process to combat foreign bodies. Phagocytes express a multicomponent enzyme complex called $\mathrm{NADPH}$ oxidase that catalyzes the electron reduction of $\mathrm{O}_{2}$. Finally, the radical $\mathrm{O}_{2} \bullet$ - can function as a molecular indicator due to its ability to oxidize - $\mathrm{SH}$ groups to disulfide bonds, which can activate and deactivate enzymes containing methionine [27].

$\mathrm{OH} \bullet$ is the most deleterious ROS to organisms. However, due to its very short half-life it can hardly be sequestered in vivo. These radicals often attack molecules by hydrogen abstraction and addition, resulting in unsaturation. $\mathrm{OH}$ - can be controlled by preventing the damage it causes or by inhibiting its formation $[15,16]$. $\mathrm{OH} \bullet$ is mainly formed by reaction of $\mathrm{H}_{2} \mathrm{O}_{2}$ with transition metals or the breakage of water molecules exposed to ionizing radiation. $\mathrm{OH} \bullet$ causes damage to DNA, RNA, proteins, lipids and cell membranes of the nucleus and mitochondria. In DNA, $\mathrm{OH} \bullet$ attacks both nitrogenous bases and deoxyribose. The attack on the sugar can be accomplished by abstraction of one of the hydrogen atoms and almost always leads to disruption of the DNA strand. The most common example of the attack on lipids is its action on the lipid membrane. OH• attacks eligible lipid chain site allyl methylenic groups, converting them into new free radical centers. The carbon radical easily adds oxygen, generating lipid peroxyl radicals that subsequently attack membrane proteins and produce damage to the cells [13].

$\mathrm{H}_{2} \mathrm{O}_{2}$ is generated in vivo by the dismutation of radical $\mathrm{O}_{2} \bullet$ - by oxidase enzymes or by $\beta$-oxidation of fatty acids. Mitochondria are important sources of radical $\mathrm{O}_{2} \bullet$ - and the presence of this anion radical can cause serious damage. They are also rich in SOD, which converts $\mathrm{O}_{2}$ - into $\mathrm{H}_{2} \mathrm{O}_{2}$. The generated $\mathrm{H}_{2} \mathrm{O}_{2}$ is then partially eliminated by CAT and GPx; however, as their effectiveness is limited, most of the $\mathrm{H}_{2} \mathrm{O}_{2}$ is released into the cell [13].

"Living organisms have adapted not only to peaceful coexistence with the free radicals, but in reality, have developed mechanisms to take advantage of them. " [13]

Despite its harmful effects, ROS play a vital role in immune cells, and without them, the body would be unprotected against infections caused by viruses, bacteria and fungi. Phagocytes and fibroblasts produce ROS during the inflammatory process to combat these infiltrating bodies. ROS from phagocytes are produced with the aid of the leukocyte membrane enzyme NADPH oxidase, which catalyzes the one electron reduction of $\mathrm{O}_{2}$ by transferring the charge to a molecule of NADPH [28]. The radical nitric oxide NO• can be produced by the action of nitric oxide synthase from arginine, oxygen and NADPH, conversion of NADPH into NADP+ also generates citrulline. This radical can be produced in greater quantities by human phagocytes when stimulated. Nitrate can be transformed into nitrite, which reacts with gastric acid to generate nitrous acid $\left(\mathrm{HNO}_{2}\right)$. Nitrous oxide $\left(\mathrm{N}_{2} \mathrm{O}_{3}\right)$ is also a precursor of nitrous acid $\left(\mathrm{HNO}_{2}\right)$ as a result of its reaction with water. Nitrous acid $\left(\mathrm{HNO}_{2}\right)$ promotes deamination of DNA bases containing free $\mathrm{NH}_{2}$ groups; for example, deamination of cytosine, adenine and guanine results in the formation of uracil, hypoxanthine and xanthine, respectively [27,29]. Nitric oxide (NO•) is not sufficiently reactive to attack DNA directly, but it can react with radical $\mathrm{O}_{2} \bullet$ - produced by phagocytes and generate peroxynitrite (ONOO-). These molecules can undergo secondary reactions, acting as agents capable of nitrating aromatic amino acids; for example, nitration of tyrosine generates nitrotyrosine and DNA bases, particularly guanine, where the main product is 8-nitroguanina [13]. The presence of $\mathrm{CO}_{2} / \mathrm{HCO}_{3}$ buffer contributes to the nitration of biomolecules because the carbonate radical to be protonated $\left(\cdot \mathrm{HCO}_{3}\right)$ forms aromatic rings.

The formation of free radicals in vivo occurs via the catalytic action of enzymes during the electron transfer processes occurring during cellular metabolism and by exposure to exogenous factors. However, the concentration of these radicals may increase due to increased intracellular generation or deficiency of antioxidant mechanisms [26-29].

Oxidative stress occurs when there is a lack of dynamic balance (or "imbalance") between the production of oxidants and the concentration of antioxidant defenses, leading to cellular damage. Oxidative stress can occur by increased production of free radicals due to a decrease in defenses or both processes simultaneously [13]. As mentioned above, oxidizing agents are formed during the normal course of metabolism; however, in certain pathological conditions, they can be produced in excess, leading to oxidative stress and possible cell death. A number of pathological processes (such as cancer and ischemia) and non-pathological processes (such as aging) are associated with oxidative stress.

\section{The antioxidant defense system}

During the process of evolution, living organisms developed efficient defense mechanisms to combat the deleterious action of ROS and to allow for life in an oxygenated environment [13]. Antioxidants are substances that are capable, even at low concentrations, of competing with other oxidizable substrates; therefore, they significantly retard or inhibit oxidation of that substrate. The main function of the human body's antioxidant defense system is to inhibit or reduce the damage caused to cells by ROS. A wide variety of antioxidants that can be classified as either enzymatic antioxidant defense systems or on-enzymatic antioxidant defense systems are explained below.

\section{Enzymatic antioxidant defense system}

This defense system consists of four major enzymes responsible for combating ROS: SOD, the GPx/ GR system and CAT. The first defense against radical $\mathrm{O}_{2} \bullet-$ is the enzyme SOD. The major isozymes of SOD are dependent on copper and zinc $(\mathrm{Cu}, \mathrm{ZnSOD})$ and a dependent (Mn-SOD). Cu-ZnSOD primarily operates in the cytosol, and MnSOD acts in the mitochondrial matrix [13]. The direct action of this enzyme is to sequester radical $\mathrm{O}_{2} \bullet-$, yielding $\mathrm{H}_{2} \mathrm{O}_{2}$ [13]. Although these three enzymes use different paths, they have the same function: to control the levels of $\mathrm{H}_{2} \mathrm{O}_{2}$ and hydroperoxides formed during the process of dismutation of radical $\mathrm{O}_{2} \bullet-$ and lipid peroxidation, respectively, through its transformation into $\mathrm{H}_{2} \mathrm{O}$ and $\mathrm{O}_{2}$ [13]. GPx and GR work together to reduce GSH and NADPH for optimal functioning of the glutathione system. Similarly, SOD, GPx and GR are distributed both in the cytosol (approximately 45\%) and mitochondria (approximately 55\%) [13]. 
The activity of GPx and CAT differ from tissue to tissue within the human body, although significant variation between the activities of these two enzymes in the skeletal muscle has not been demonstrated. Although GPx in combination with GR acts both in the cytosol and mitochondria, CAT is mainly present in organelles called peroxisomes. Peroxisomes primarily control $\mathrm{H}_{2} \mathrm{O}_{2}$ levels. Although uncertainties exist regarding whether these two enzymes perform the same function within the same cell, it is known that the affinity of $\mathrm{GPx}$ by $\mathrm{H}_{2} \mathrm{O}_{2}$ is approximately 10 times higher than that of CAT [13]. Therefore, at low concentrations of $\mathrm{H}_{2} \mathrm{O}_{2}$, GPx is largely responsible for controlling levels of intracellular peroxide, with CAT more actively contributing as $\mathrm{H}_{2} \mathrm{O}_{2}$ concentration increases within the cell.

Another interesting feature of this system is the intramuscular distribution of these enzymes: the more oxidative muscle fibers (type I) possess greater amounts of all antioxidant enzymes, but the more glycolytic fibers (type II) contain them in smaller amounts $[15,16]$.

\section{Non-enzymatic antioxidant defense system}

The other major group of antioxidant defenses is the enzymatic system, also known as "protection through small molecules". Among the major antioxidant molecules are vitamins $\mathrm{A}, \mathrm{E}$ and $\mathrm{C}$, as well as significantly reduced glutathione that acts as a potent antioxidant. These antioxidant molecules assist with the detoxification of ROS by donating their electron molecules to the newly formed free radical molecules without turning into reactive radicals $[15,16]$. Vitamins act in different intracellular compartments. With soluble characteristics, vitamins $\mathrm{A}$ and $\mathrm{E}$ are located inside the cell membranes, whereas water-soluble vitamin $\mathrm{C}$ acts in the cytosol and participates in the reoxidation of fat soluble vitamins $[15,16]$. Reduced glutathione (GR) is a tripetide containing a thiol grouping and is found in almost all animal and plant cells $[15,16]$. Its main role is that of a substrate for GPx; however, it still operates in a similar way to vitamin C.

\section{Exercise and ROS production (Oxidative stress)}

Studies have shown that intense exercise causes oxidative stress in animals and humans. This may be related to chronic fatigue syndrome $[1,17]$, delayed onset muscle soreness and tissue lesions [30-33]. These concerns are of utmost importance for understanding exercise. Exercise induces an increase in oxygen consumption and energy demand by inducing increased production of ROS [2-12]. The increased production of ROS associated with the inefficiency of neutralization by the antioxidant defense system can lead to oxidative stress. Under these circumstances, ROS reacts with cellular structures, inducing high levels of oxidation by altering the function and impairing intracellular homeostasis. The molecular mechanism involved in oxidative stress and muscle damage induced by an acute exercise protocol has been the subject of intense investigation in recent years [32-34]. Oxygen consumption in the human body is increased many times when an individual is exercising at high intensities [36-38]. The oxygen utilization by skeletal muscles during intense physical exertion may increase approximately 15 to 20 -fold compared with consumption at rest [35-37]. This increased muscle metabolism leads to an increase in the flow of electrons due to the growth rate of mitochondrial respiration in the active muscle. Furthermore, increased muscle metabolism can lead to increased leakage of electrons and, consequently, increased formation of ROS [35-37]. Both aerobic and anaerobic exercise can promote important functional morphology and metabolic adaptations in the body, although this practice is also related to excessive production of ROS [2-7]. ROS can be produced by different mechanisms during physical exercise, including the partial reduction of oxygen in the mitochondria (electron transport chain) and the inflammatory process [29-33]. Inflammation is a major source of ROS during and after physical exercise because intense contractions cause injury to the muscle fibers, which require removal of damaged proteins followed by re-synthesis of new proteins. Aerobic exercise causes increased flow of oxygen in the mitochondria. Because two to five percent of this oxygen is not completely reduced, ROS are formed [29-33]. Moreover, although anaerobic exercise can be performed independent of the oxygen supply, excessive production of ROS has been observed during this type of stress [29-33] likely due to other mechanisms. It should also be noted that under anaerobic conditions, metabolic stress increases the degradation of adenosine triphosphate (ATP), activating the xanthine oxidase pathway (XO) and yielding additional production of $\mathrm{O}_{2} \bullet$ - radicals [29-34].

The scientific literature has reported two major sources of ROS production during and after exercise: the electron transport chain (ETC) of mitochondria and NADPH oxidization pathways [35].

\section{Electron transport chain}

Oxygen belongs to family VI in the periodic table of elements, with an atomic number and atomic mass of 8 and 16.0, respectively. Thus, oxygen possesses 8 electrons distributed in its electron shells. Because of its configuration, oxygen molecules tend to accept one electron at a time, forming highly reactive intermediates [13]. Typically, approximately 95 to $98 \%$ of oxygen absorbed by aerobic organisms is reduced, forming water. This occurs in the respiratory chain of mitochondria through electron transport as well as in the endoplasmic reticulum, where the enzyme cytochrome oxidase induces tetravalent reduction of $\mathrm{O}_{2}$ via the process of oxidative phosphorylation. NADH, FADH and the ubiquinone or coenzyme Q serve as sources for the hydrogen cation and electrons for this reaction [13]. However, as already stated, 2 to $5 \%$ of $\mathrm{O}_{2}$ is univalently reduced during the process in which the molecule receives only one electron that will occupy one of the external orbitals while the other remains unpaired, producing ROS that can become free radicals under the processes described earlier. The monovalent reduction (one electron at a time) of oxygen in the electron carrier chain generates the formation of ROS.

\section{Via NADPH oxidase}

NADPH oxidase is a multi-enzyme complex composed of the subunits gp91phox in the membrane, $\mathrm{p}^{22}$ phox (which together form the flavocitocromo B558) and cytosolic subunits, such as $\mathrm{p}^{47}$ phox, $\mathrm{p}^{60}$ phox, $\mathrm{p}^{67}$ phox and Rac1 or Rac2. NADPH oxidase is present in leucocytes, endothelial cells and skeletal muscle [38,39]. When activated, some subunits are phosphorylated by different kinases such as protein kinase $\mathrm{C}(\mathrm{PKC})$ and translocated to the membrane, forming the catalytically active oxidase $[36,37]$. The route of NADPH oxidasedependent PKC activation is involved in mechanisms responsible for oxidative stress that affect vascular cells. Moreover, hyperglycemia has been reported to be primarily responsible for the activation of this pathway $[13,40]$. NADPH oxidase is cited as the main source for the production of ROS in blood vessels and may be associated with numerous pathophysiological processes of various diseases, including hypercholesterolemia, atherosclerosis and hypertension [40,41]. High glucose levels lead to increased de novo synthesis of DAG (Diacylglycerol), the physiological activator of Protein Kinase C (PKC), which increases the activity of NADPH oxidase and thus results in increased production of radical $\mathrm{O}_{2} \bullet-[39,40]$. Recent studies 
indicate that phagocytic NADPH oxidase type is the major source of ROS production in many non-phagocytic cells, including fibroblasts, smooth muscle cells, endothelial cells, renal mesangial cells and renal tubular cells. This enzyme system was originally discovered in neutrophils, which are potential sources of large amounts of radical $\mathrm{O}_{2} \bullet$ - during phagocytosis and are vital to innate defense $[39,40]$. The ROS production by oxidase in small amounts can function in signaling and metabolic pathways; in large quantities, it can cause oxidative damage. Cell types found in vessel walls generally have similar phagocytic NADPH oxidases that are activated under physiological conditions. These oxidases appear to have multiple functions, including controlling vascular function, responses to gene expression and signaling in numerous cellular processes such as growth, apoptosis, migration and remodeling of the extracellular matrix.

\section{Discussion}

Exercise is increasingly present in the lives of the human population and is the primary requirement for the maintenance and promotion of health [1]; however, many studies have shown that intense exercise increases the production of ROS [2-8]. If this increase is not accompanied by an adaptation of the antioxidant defense system, it is possible to install a framework known as oxidative stress that is directly related to several chronic diseases, such as diabetes, cancer, atherosclerosis and neurodegenerative diseases including Parkinson's and Alzheimer's [14,15]. However a growing body of evidence has suggested that increased ROS production plays an important role in muscle metabolism, in both homeostasis and the adaptive response to exercise $[15,16]$. Recent results have shown that ROS may not merely represent random generators of the destruction of cellular structures but that at certain levels (at physiological concentrations or perhaps at levels above physiological levels), they are beneficial to the cell by actively participating in pathways that regulate growth, differentiation and cell proliferation [13-16]. This specific signaling may be crucial for adaptive responses induced by physical exercise. In this sense, acute exercise increases the production of ROS in the muscle tissue, which can act as messengers [13-16].

Another research question worth investigation is whether the ROS signal in tissue remodeling can be induced by the exercise process. One possible explanation is that vigorous exercise triggers muscular ROS production that may activate nuclear factor redox activity and induce intracellular stimulus to protein synthesis [13-16]. Another possible explanation is that ROS can coordinate leukocyte (neutrophil and monocyte) influx to the skeletal muscle tissue, and this infiltration plays an important role in an adaptive muscle process [40-51]. Using a pharmacological antioxidant strategy (apocynin) and mutant animal model (gp91phox-/-), it is possible to block this process and possibly interrupt remodeling of tissues following exercise. These results show that ROS from NADPH oxidation play an important role in leukocyte recruitment and that blockage/deletion of NADPH-oxidase may inhibit rolling, adhesion and transmigration [45]. Currently, it is not possible to determine the source of ROS in this system, but ROS may be produced through endothelial cells, skeletal muscle cells and leukocytes following the expression of NADPH-oxidase with the gp91phox subunit [45].

A balance between the production of reactive species and the antioxidant defense systems appears to represent a central component in many adaptive responses in skeletal muscle, as well as hypertrophy and atrophy. If the production of ROS is ideal (a bit higher than basal levels), an adaptive response can result in addition to hypertrophy, angiogenesis and mitochondrial biogenesis; however, if ROS levels are increased significantly above basal levels and the antioxidant defense capacity, there is an atrophic response and damage to different organs, such as is observed with Duchenne muscular dystrophy in skeletal muscle tissue [31]. James Watson, an important American researcher, who was awarded the Nobel Prize in Physiology and Medicine in 1962, published an article in The Lancet [52] that argued that ROS produced by exercise play a role in several important adaptive responses to promote the health of the individual. Recent studies published in respected journals claim that the administration of antioxidants could compromise the benefits of physical exercise [53]. Therefore, the conflict between ROS production and the antioxidant defense system represents a central issue in the adaptive response of muscle tissue that is important for aspects of sports performance, such as angiogenesis, mitochondrial biogenesis and muscle hypertrophy. There is a large body of literature that describes how inflammation is an exercisedinduced physiological response that happens occurs after acute episodes of exercise and is capable of chronically inducing the adaptation of skeletal muscle tissue after a period of training time. It is now becoming clear that ROS play an important role in the acute inflammatory process after intense exercise [45] and that the skeletal muscle inflammatory process may play a role in the skeletal muscle adaptive response [41-45]. In this sense, anti-oxidant administration as a supplemental diet aid could inhibit these important adaptive responses and block the benefits of exercise from recreational levels and high levels of exercise training. Based on our results from the laboratory and a recent literature review, we have developed a hypothesis that may represent the association between intense exercise and increased production of ROS in skeletal muscle tissue (Figure 1). In this figure, we summarized the possible role played by ROS during leukocyte migration into muscle tissue resulting from exercise. Vigorous exercise triggers ROS production in skeletal muscle that can help the migration of leukocytes and activate signaling pathways. Nunes-Silva et al. [45] has shown that drugs or genetic deletion of NADPH-oxidase inhibited rolling, adhesion and transmigration of leukocytes. It is not possible to determine the source of ROS in this system, but endothelial cells, skeletal muscle cells and leukocytes may produce ROS because these cells express NOX-2, the NADPH-oxidase that contains the gp91phox subunit. The results from this work also showed enhancement of the number of leukocytes adherent and transmigrating when $\mathrm{SOD}$ is used, suggesting that $\mathrm{H}_{2} \mathrm{O}_{2}$ is the probable ROS molecule involved. One potential function of ROS may be to recruit cells to the exercised muscle and orchestrate the resolution of the function of the damaged tissue and induce the proper adaptive response to exercise training. ROS-mediated infiltration of neutrophils after exercise may also contribute to exercise remodeling processes, including angiogenesis, the hypertrophic response and mitochondrial biogenesis [9]. Taken together, our data suggest that exercise-triggered ROS production enhanced leukocyte-endothelium interactions that coordinate neutrophil infiltration to the exercised skeletal muscle. Finally, recent findings do not offer a consensus to support diet supplementation of anti-oxidant drugs to help in the exercise scenario. 


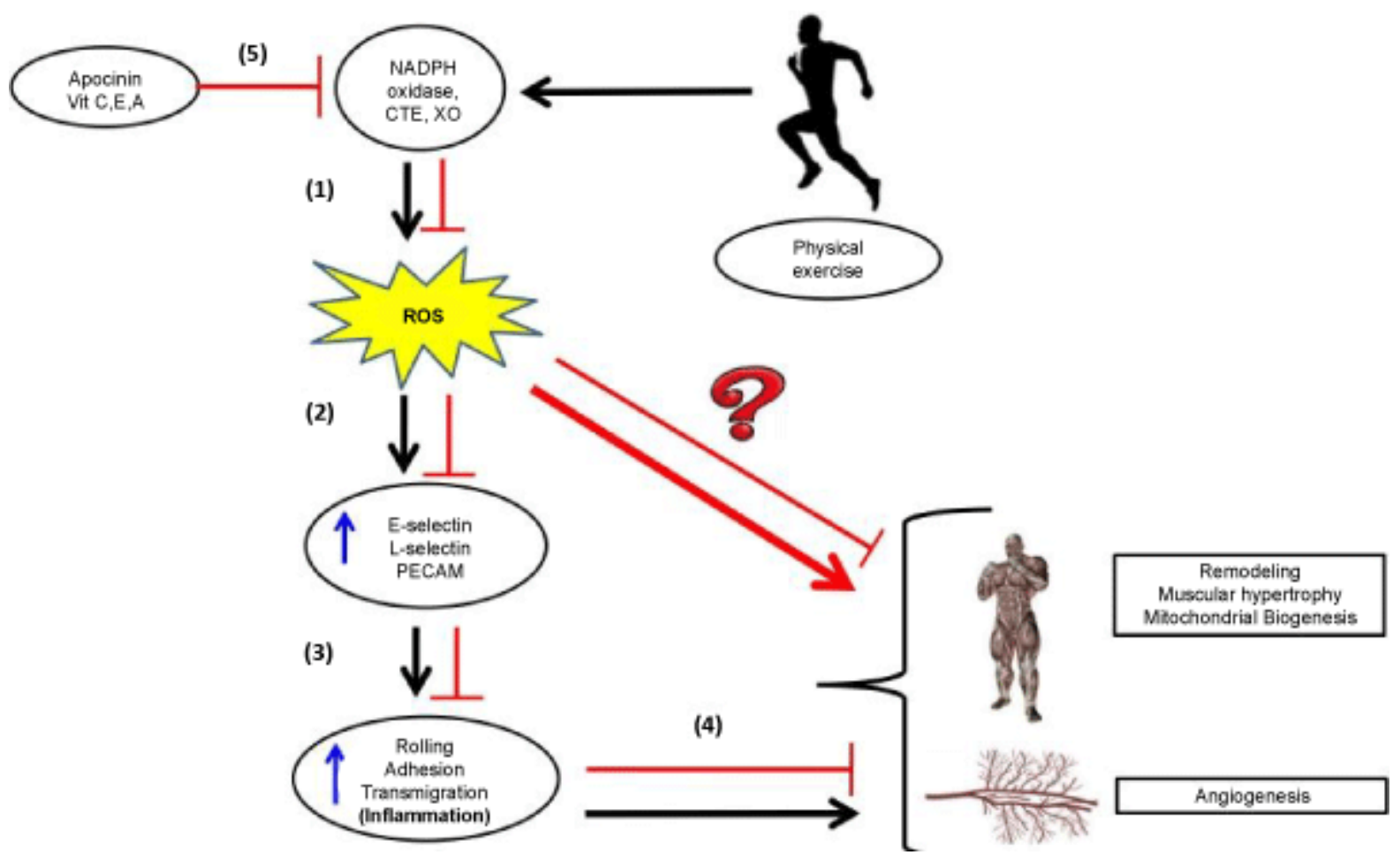

Figure 1: Interaction between exercise, oxidative stress and skeletal muscle adaptive response.

(1) Exercise performed in an appropriate work load (high intensity and sufficient duration) alters homeostasis and increases metabolism, thus producing a greater amount of (ROS) by direct activated pathways such as the electron transport chain and indirect pathways such as Xanthine Oxidase (XO) and NADPH oxidase.

(2) This increased production of ROS activates intracellular signaling pathways that culminate in increased surface adhesion molecules on the vascular endothelium and leukocytes.

(3) The increase in adhesion molecules enables higher leukocyteendothelial interaction as represented by the increased number of cells in the rolling process or adhered to post-capillary venules. In the presence of tissue damage, these cells can then transmigrate from the muscle interstitium toward the existing lesion.

(4) We propose that these acute events due to increased ROS production result in a local inflammatory process within the skeletal muscle tissue that contributes to remodeling of the tissue, generating an "up regulation" that prepares the system for increasing muscle tissue work.

(5) Therefore, the inhibition or blockage of ROS production with antioxidant supplementation could inhibit the adaptive response for chronic practice (training) exercise.

\section{Conclusion}

This literature investigation demonstrates that there is increased production of ROS during and after the completion of high-intensity exercise in sites including NADPH oxidase. This elevated production may not pose a risk to the health of the practitioner and may also represent a signaling adaptation response to exercise. Nevertheless, this review shows that ROS generated in response to exercise might be important signaling molecules rather than solely danger agent.

\section{References}

1. Berryman JW (2010) Exercise is medicine: a historical perspective. Curr Sports Med Rep 9: 195-201.

2. Davies KJ, Quintanilha AT, Brooks GA, Packer L (1982) Free radical and tissue damage produced by exercise. Biochem Biophys Res Commun 107: 1198-1205.

3. Vollaard NB, Shearman JP, Cooper CE (2005) Exercise-induced oxidative stress:myths, realities and physiological relevance. Sports Med 35: 1045-1062.

4. Temiz A, Başkurt OK, Pekçetin C, Kandemir F, Güre A (2000) Leukocyte activation, oxidant stress and red blood cell properties after acute, exhausting exercise in rats. Clin Hemorheol Microcirc 22: 253-259.

5. Alessio HM (1993) Exercise-induced oxidative stress. Med Sci Sports Exerc 25: 218-224.

6. Barbieri E, Sestili P (2012) Reactive oxygen species in skeletal muscle signaling. J Signal Transduct 2012: 982794.

7. Close GL, Ashton T, Cable T, Doran D, MacLaren DP (2004) Eccentric exercise, isokinetic muscle torque and delayed onset muscle soreness: the role of reactive oxygen species. Eur J Appl Physiol 91: 615-621.

8. Cooper CE, Vollaard NB, Choueiri T, Wilson MT (2002) Exercise, free radicals and oxidative stress. Biochem Soc Trans 30: 280-285.

9. Gomes EC, Silva AN, de Oliveira MR (2012) Oxidants, antioxidants, and the beneficial roles of exercise-induced production of reactive species. Oxid Med Cell Longev 2012: 756132.

10. Ji LL (1993) Antioxidant enzyme response to exercise and aging. Med Sci Sports Exerc 25: 225-231. 
11. Nikolaidis MG, Jamurtas AZ (2009) Blood as a reactive species generator and redox status regulator during exercise. Arch Biochem Biophys 490: 77-84.

12. Lee J, Goldfarb AH, Rescino MH, Hegde S, Patrick S, et al. (2002) Eccentric exercise effect on blood oxidative-stress markers and delayde onset of muscle soreness. Med Sci Sports Exerc 34: 443-448.

13. Dröge W (2002) Free radicals in the physiological control of cell function. Physiol Rev 82: 47-95.

14. Powers SK, Jackson MJ (2008) Exercise-induced oxidative stress: cellular mechanisms and impact on muscle force production. Physiol Rev 88: 1243-1276.

15. Powers SK, Talbert EE, Adhihetty PJ (2011) Reactive oxygen and nitrogen species as intracellular signals in skeletal muscle. J Physiol 589: 2129-2138.

16. Pattwell DM1, McArdle A, Morgan JE, Patridge TA, Jackson MJ (2004) Release of reactive oxygen and nitrogen species from contracting skeletal muscle cells. Free Radic Biol Med 37: 1064-1072.

17. Kaneko T, Qingyou X, Miyamoto Y, Shengyu M, Wang H, et al. (2013) Oxidative Stress in Multiple Organ Damage in Hypertension, Diabetes and CKD, Mechanisms and New Therapeutic Possibilities. Intech.

18. Blair SN, Kohl HW 3rd, Barlow CE, Paffenbarger RS Jr, Gibbons LW, et al. (1995) Changes in physical fitness and all-cause mortality. A prospective study of healthy and unhealthy men. JAMA 273: 1093-1098.

19. Moore SC, Patel AV, Matthews CE, Berrington de Gonzalez A, Park Y, et al. (2012) Leisure Time Physical Activity of Moderate to Vigorous Intensity and Mortality: A Large Pooled Cohort Analysis. PLoS Med 9: e1001335.

20. Lee IM, Shiroma EJ, Lobelo F, Puska P, Blair SN, et al. (2012) Effect of physical inactivity on major non-communicable diseases worldwide: an analysis of burden of disease and life expectancy. Lancet 380: 219-229.

21. Kakanis MW, Peake J, Brenu EW, Simmonds M, Gray B, (2010). The open window of susceptibility to infection after acute exercise in healthy young male elite athletes. Exerc Immunol Rev 16: 119-137.

22. Nieman DC, Pedersen BK (1999) Exercise and immune function. Recent developments. Sports Med 27: 73-80.

23. Pyne DB, Gleeson M (1998) Effects of intensive exercise training on immunity in athletes. Int J Sports Med 19 Suppl 3: S183-S191.

24. Pedersen BK, Toft AD (2000) Effects of exercise on lymphocytes and cytokines. Br J Sports Med 34: 246-251.

25. Pedersen BK, Hoffman-Goetz L (2000) Exercise and the immune system: regulation, integration, and adaptation. Physiol Rev 80: 1055-1081.

26. Frey RS, Ushio-Fukai M, Malik AB (2009) NADPH oxidase-dependent signaling in endothelial cells: role in physiology and pathophysiology. Antioxid Redox Signal 11: 791-810.

27. Jiang F, Zhang Y, Dusting GJ (2011) NADPH oxidase-mediated redox signaling: roles in cellular stress response, stress tolerance, and tissue repair. Pharmacol Rev 63: 218-242.

28. El-Benna J, Dang PM, Gougerot-Pocidalo MA, Elbim C (2005) Phagocyte NADPH oxidase: a multicomponent enzyme essential for host defenses. Arch Immunol Ther Exp (Warsz) 53: 199-206.

29. Babior BM (1999) NADPH oxidase: an update. Blood 93: 1464-1476.

30. Cheung K, Hume P, Maxwell L (2003) Delayed onset muscle soreness : treatment strategies and performance factors. Sports Med 33: 145-164.

31. Tidball JG (2005) Inflammatory processes in muscle injury and repair. Am J Physiol Regul Integr Comp Physiol 288: R345-R353.

32. Peake JM, Suzuki K, Wilson G, Hordern M, Nosaka K, et al. (2005) Exercise-induced muscle damage, plasma cytokines, and markers of neutrophil activation. Med Sci Sports Exerc 37: 737-745.
33. Peake JM, Suzuki K, Coombes JS (2007) The influence of antioxidant supplementation on markers of inflammation and the relationship to oxidative stress after exercise. J Nutr Biochem 18: 357-371.

34. Peake J, Nosaka K, Suzuki K (2005) Characterization of inflammatory responses to eccentric exercise in humans. Exerc Immunol Rev 11: 64-85.

35. Ferreira JC, Bacurau AV, Bueno CR Jr, Cunha TC, Tanaka LY, et al. (2010) Aerobic exercise training improves $\mathrm{Ca} 2+$ handling and redox status of skeletal muscle in mice. Exp Biol Med (Maywood) 235: 497-505.

36. Ferreira JC, Rolim NP, Bartholomeu JB, Gobatto CA, Kokubun E, et al. (2007) Maximal lactate steady state in running mice: effects of exercise training. Clin Exp Pharmacol Physiol 34: 760-765.

37. Nikolaidis MG, Kyparos A, Spanou C, Paschalis V, Theodorou AA, et al. (2012) Redox biology of exercise: an integrative and comparative consideration of some overlooked issues. J Exp Biol 215: 1615-1625.

38. Ray R, Shah AM (2005) NADPH oxidase and endothelial cell function. Clin Sci (Lond) 109: 217-226.

39. Whitehead NP, Yeung EW, Froehner SC, Allen DG (2010) Skeletal muscle NADPH oxidase is increased and triggers stretch-induced damage in the mdx mouse. PLoS One 5: e15354.

40. Frey RS, Ushio-Fukai M, Malik AB (2009) NADPH oxidase-dependent signaling in endothelial cells: role in physiology and pathophysiology. Antioxid Redox Signal 11: 791-810.

41. Schneider BS, Tiidus PM (2007) Neutrophil infiltration in exerciseinjured skeletal muscle: how do we resolve the controversy? Sports Med 37: 837-856.

42. Smith C, Kruger MJ, Smith RM, Myburgh KH (2008) The Inflammatory Response to Skeletal Muscle Injury Illuminating Complexities. Sports Med 38: 947-969.

43. Butterfield TA, Best TM, Merrick MA (2006) The dual roles of neutrophils and macrophages in inflammation: a critical balance between tissue damage and repair. J Athl Train 41: 457-465.

44. Nozawa H, Chiu C, Hanahan D (2006) Infiltrating neutrophils mediate the initial angiogenic switch in a mouse model of multistage carcinogenesis. Proc Natl Acad Sci U S A 103: 12493-12498.

45. Nunes-Silva A, Bernardes PT, Rezende BM, Lopes F, Gomes EC, et al. (2014) Treadmill exercise induces neutrophil recruitment into muscle tissue in a reactive oxygen species-dependent manner. An intravital microscopy study. PLoS One. 9: e96464.

46. Chen GY, Nuñez G (2010) Sterile inflammation: sensing and reacting to damage. Nat Rev Immunol 10: 826-837.

47. Kolaczkowska E, Kubes P (2013) Neutrophil recruitment and function in health and inflammation. Nat Rev Immunol 13: 159-175.

48. Kubes P, Kerfoot SM (2001) Leukocyte recruitment in the microcirculation: the rolling paradigm revisited. News Physiol Sci 16: 76-80.

49. Kubes P, Mehal WZ (2012) Sterile inflammation in the liver. Gastroenterology 143: 1158-1172.

50. Ley K, Laudanna C, Cybulsky MI, Nourshargh S (2007) Getting to the site of inflammation: the leukocyte adhesion cascade updated. Nat Rev Immunol 7: 678-689.

51. Medzhitov R (2008) Origin and physiological roles of inflammation. Nature 454: 428-435.

52. Watson JD (2014) Type 2 diabetes as a redox disease. Lancet 383: 841-843.

53. Ristow M, Zarse K, Oberbach A, Klöting N, Birringer M, et al. (2009) Antioxidants prevent health-promoting effects of physical exercise in humans. Proc Natl Acad Sci U S A 106: 8665-8670. 